\title{
The neural basis of video gaming
}

\author{
S Kühn ${ }^{1,2,3}$, A Romanowski ${ }^{2}$, C Schilling ${ }^{2}$, R Lorenz ${ }^{2}$, C Mörsen ${ }^{2}$, N Seiferth ${ }^{2}$, T Banaschewski ${ }^{4}$, A Barbot ${ }^{5}$, GJ Barker ${ }^{6}$, C Büchel ${ }^{7}$, \\ PJ Conrod ${ }^{6}$, JW Dalley ${ }^{8,9}$, H Flor $^{10}$, H Garavan ${ }^{11}$, B Ittermann ${ }^{3}$, K Mann ${ }^{12}$, J-L Martinot ${ }^{13,14}$, T Paus ${ }^{15,16,17}$, M Rietschel ${ }^{18}$, \\ MN Smolka ${ }^{19,20}$, A Ströhle ${ }^{1}$, B Walaszek ${ }^{3}$, G Schumann ${ }^{6}$, A Heinz $^{2}$, J Gallinat ${ }^{2}$ and The IMAGEN Consortium
}

\begin{abstract}
Video game playing is a frequent recreational activity. Previous studies have reported an involvement of dopamine-related ventral striatum. However, structural brain correlates of video game playing have not been investigated. On magnetic resonance imaging scans of 154 14-year-olds, we computed voxel-based morphometry to explore differences between frequent and infrequent video game players. Moreover, we assessed the Monetary Incentive Delay (MID) task during functional magnetic resonance imaging and the Cambridge Gambling Task (CGT). We found higher left striatal grey matter volume when comparing frequent against infrequent video game players that was negatively correlated with deliberation time in CGT. Within the same region, we found an activity difference in MID task: frequent compared with infrequent video game players showed enhanced activity during feedback of loss compared with no loss. This activity was likewise negatively correlated with deliberation time. The association of video game playing with higher left ventral striatum volume could reflect altered reward processing and represent adaptive neural plasticity.
\end{abstract}

Translational Psychiatry (2011) 1, e53; doi:10.1038/tp.2011.53; published online 15 November 2011

\section{Introduction}

Video and computer games have become a highly popular spare-time activity for children, adolescents as well as adults. The literature reports favourable and adverse effects of frequent video game playing. It has been demonstrated that video game playing can enhance visual skills related to attention ${ }^{1,2}$ and probabilistic inferences. ${ }^{3}$ Furthermore, improvements in higher cognitive executive functions such as task switching, working memory and reasoning have been associated with gaming improvements in older adults. ${ }^{4}$

Recently, the neural processes underlying video game playing and gambling have been studied with functional neuroimaging. Several studies have implicated an involvement of the brain reward system in gaming and computer gambling. By means of positron emission tomography, increased release of dopamine in the ventral striatum while video gaming and a positive correlation with the performance has been reported in healthy subjects. ${ }^{5}$ Using functional magnetic resonance imaging (fMRI), performance of healthy volunteers in the lowa gambling task has been associated with increases in blood-oxygen-level-dependent (BOLD) activity of the ventral striatum. ${ }^{6}$ Activation of the dorsal striatum during initial training predicted later learning success in video gaming. ${ }^{7}$

These striatum-associated findings in healthy subjects are in line with the clinical observation that dopaminergic medication in Parkinson's patients can lead to pathological gambling and other addictive behaviour such as binge eating and hypersexuality. ${ }^{8}$ Greater dopamine release in the ventral striatum has been shown in Parkinson's patients with addiction, obsession and gambling compared with Parkinson's patients without these symptoms. ${ }^{9}$ These findings identify striatal function driven by dopamine as a core candidate promoting addictive behaviour. Of note, it has recently been demonstrated that pathological gamblers have an increased striatal dopamine release while loosing money, ${ }^{10}$ a biological signal that may hinder the termination of gambling.

There is a lack of studies focussing on structural correlates of frequent video game playing. Based on previous functional neuroimaging studies stressing the involvement of the reward network in video gaming and, in particular, the

\footnotetext{
${ }^{1}$ Faculty of Psychology and Educational Sciences, Department of Experimental Psychology, Ghent Institute for Functional and Metabolic Imaging, Ghent University, Ghent, Belgium; ${ }^{2}$ Charité University Medicine, St Hedwig Krankenhaus, Clinic for Psychiatry and Psychotherapy, Campus Mitte, Berlin, Germany; ${ }^{3}$ PhysikalischTechnische Bundesanstalt (PTB), Berlin and Braunschweig, Germany; ${ }^{4}$ Department of Child and Adolescent Psychiatry, Central Institute of Mental Health, Mannheim, Germany; ${ }^{5}$ Neurospin, Commissariat à l'Energie Atomique, Paris, France; ${ }^{6}$ Kings College London, Institute of Psychiatry, London, UK; ${ }^{7}$ Department of Systems Neuroscience, University Clinic Hamburg Eppendorf, Universitaetsklinikum Hamburg Eppendorf, Hamburg, Germany; ${ }^{8}$ Department of Psychiatry, University of Cambridge, Cambridge, UK; ${ }^{9}$ Department of Experimental Psychology, Behavioural and Clinical Neurosciences Institute, University of Cambridge, Cambridge, UK; ${ }^{10}$ Department of Cognitive and Clinical Neuroscience, Central Institute of Mental Health, Mannheim, Germany; ${ }^{11}$ Institute of Neuroscience, Trinity College Dublin, Dublin, Ireland; ${ }^{2}$ Department of Addictive Behaviour and Addiction Medicine, Central Institute of Mental Health, Mannheim, Germany; ${ }^{13}$ Institut National de la Santé et de la Recherche Médicale, INSERM Unit 1000 'Imaging \& Psychiatry', University Paris Sud, Orsay, France; ${ }^{14}$ AP-HP Department of Adolescent Psychopathology and Medicine, Maison de Solenn, University Paris Descartes, Paris, France; ${ }^{15}$ Rotman Research Institute, University of Toronto, Toronto, Canada; ${ }^{16}$ Montreal Neurological Institute, McGill University, Montreal, Canada; ${ }^{17}$ School of Psychology, University of Nottingham, Nottingham, UK; ${ }^{18}$ Department of Genetic Epidemiology in Psychiatry, Central Institute of Mental Health, Mannheim, Germany; ${ }^{19}$ Department of Psychiatry and Psychotherapy, Technische Universität Dresden, Dresden, Germany and ${ }^{20}$ Neuroimaging Center, Department of Psychology, Technische Universität Dresden, Dresden, Germany

Correspondence: Dr S Kühn, Faculty of Psychology and Educational Sciences, Department of Experimental Psychology, Ghent Institute for Functional and Metabolic Imaging, Ghent University, Henri Dunantlaan 2, 9000 Gent, Belgium. E-mail: simone.kuhn@ugent.be

Keywords: gambling; nucleus accumbens; reward; video gaming; voxel-based morphometry
}

Received 30 August 2011; revised 7 October 2011; accepted 8 October 2011 
ventral striatum, we predicted volumetric differences between frequent and moderate video gamers in reward-related brain regions. Furthermore, we predicted differences in neuronal reward processing in the $\mathrm{fMRI}$ and in operationalized assessed gambling behaviour. Based on findings in pathological gambling, ${ }^{10}$ we predicted higher ventral striatum activity during feedback of loss in frequent video gamers.

We tested 154 14-year-old adolescents from the IMAGEN project ${ }^{11}$ including a questionnaire assessing video gaming frequency, structural magnetic resonance imaging scan, the Monetary Incentive Delay (MID) task ${ }^{12}$ in $\mathrm{fMRI}$ and the Cambridge Gambling Task (CGT $\left.{ }^{13}\right)$. During the MID task, participants see cues that indicate that they may win or not win money, then wait for a variable anticipatory delay period, and finally respond to a rapidly presented target with a button press to try to either win or avoid losing money. During the CGT, participants made simple probabilistic judgment between two mutually exclusive outcomes, and then placed a bet on their confidence in that decision (details in the Supplementary Material).

\section{Methods}

Participants. A total of 154 healthy 14-year-old adolescents (mean $=14.4$, s.d. $=0.32 ; 72$ males, 82 females) were recruited within the scope of the IMAGEN project, a European multicenter genetic-neuroimaging study in adolescence. ${ }^{11}$ Written informed consent was obtained from all participants as well as from their legal guardians. The adolescents were recruited from secondary schools in Berlin. The assessment was approved by local ethics committee and the head teachers of the school. Participants with a medical condition such as a tumour, neurological disorders, epilepsy, or mental-health disorders, were excluded. All participating subjects were assessed by means of self-rating and two external ratings (by their parents and a psychiatrist specialized in paediatrics) based on International Classification of Disease-10 as well as Diagnostic and Statistical Manual of Mental Disorders (The Development and Well-Being Assessment Interview, DAWBA $^{14}$ ).

Questionnaire and tasks. We administered a questionnaire exclusively in the Berlin sample assessing computer gaming behaviour (CSV-S ${ }^{15}$ ) comprising the questions: 'How many hours do you play video games on average on a weekday?' and 'How many hours do you play video games on average on a day during the weekend?'. Based on the hours indicated, we calculated the weekly hours spend on playing video games and divided the group of participants by the median of $9 \mathrm{~h}$ into frequent ( $n=76: 24$ female, 52 male) and infrequent video game players ( $n=78$ : 58 female, 20 male).

During $\mathrm{fMRI}$, participants performed the Monetary Incentive Delay (MID) task. ${ }^{12}$ The MID task is a reaction time task that has been used to assess brain activity during reward anticipation and reward feedback. In each of the 66 trials of $10 \mathrm{~s}$ duration, participants first saw one of three visual cues (250 ms) denoting whether a target (white square) would subsequently appear on the left or right side of the screen and whether participants could win 0,2 or 10 points in this trial.
After a variable delay (4000-4500 ms), participants were asked to respond with a left or right button press as soon as the target was presented $(100-300 \mathrm{~ms})$ on the left or right side of the screen. Anticipating button presses or button presses after target presentation or wrong button presses, resulted in no gain. Feedback on how many points were won during the trial was presented for $1450 \mathrm{~ms}$ after the response. Task difficulty, namely target duration was individually adjusted so that each participant succeeded on about two-thirds of all trials. Before scanning, participants completed a practice session of $5 \mathrm{~min}$ duration (for more details see Knutson et al. ${ }^{12}$.

Moreover, we administrated an adaptation of the $\mathrm{CGT}^{13}$ outside of the scanner, in which subjects made a simple probabilistic judgment between two mutually exclusive outcomes, and then placed a bet on their confidence in that decision. On each trial, the subject was presented with a mixture of 10 red and blue boxes, and had to guess the colour of the box that hides a single yellow token. The ratio of coloured boxes varied across 9:1, 8:2, 7:3 and 6:4 on a trial-totrial basis, in a randomized manner. Token location was pseudo-randomized and independent on each trial. Hence, on a 9:1 trial, the probability was 90:10. Then subjects indicated their decision by touching a response panel labelled 'red' or 'blue' on a touch screen. The subjects were then asked to make a bet on the confidence in their decision, in order to increase the point score over trials. Possible bets were presented in either an ascending or descending sequence of $5,25,50,75$ and $95 \%$ of the points held at the time of the decision. Each bet was presented for $2 \mathrm{~s}$ before being replaced by the next bet. Subjects first completed 36 trials with the bets presented in an ascending sequence, and then 36 in a descending sequence, counterbalanced for order across subjects. After betting, feedback was provided and the position of the yellow token was shown. The amount of the bet was either added or subtracted to the subject's total score. Usually, three dependent variables are derived from the CGT: the latency to make a decision, the proportion of trials on which the subject chooses the more likely box colour, and the percentage of points bet on each decision.

Scanning procedure. Structural images were collected on a General Electric 3T scanner (GE Signa EXCITE, Milwaukee, WI, USA) and a Siemens Verio 3T (Siemens, Erlangen, Germany) with a standard eight-channel head coil. The participants measured on the GE scanner consisted of 35 frequent and 30 infrequent video gamers and 41 frequent and 48 infrequent video gamers measured on the Siemens scanner $\left(\chi^{2}=0.91, P=0.42\right)$. The images were obtained using a three-dimensional T1-weighted magnetization-prepared gradient-echo sequence (MPRAGE) based on the ADNI protocol (http://www.adni-info.org; GE scanner: repetition time $=7.16 \mathrm{~ms}$; echo time $=3.02 \mathrm{~ms}$; flip angle $=8^{\circ} ; 256 \times 256 \times 166$ matrix, $1.1 \times 1.1 \times 1.1 \mathrm{~mm}^{3}$ voxel size; Siemens scanner: repetition time $=6.9 \mathrm{~ms}$; echo time $=2.93 \mathrm{~ms} ;$ flip angle $=9^{\circ} ; 240 \times 256 \times 160$ matrix, $1.1 \times 1.1 \times 1.1 \mathrm{~mm}^{3}$ voxel size). Whole-brain functional images were collected on the same scanners using a T2*-weighted echo planar imaging (EPI) sequence sensitive to BOLD contrast (time of repetition $(T R)=2200 \mathrm{~ms}$, 
echo time $(T E)=30 \mathrm{~ms}$, image matrix $=64 \times 64$, field of view $(\mathrm{FOV})=224 \mathrm{~mm}$, flip angle $=80^{\circ}$, slice thickness $=2.4 \mathrm{~mm}$, $1 \mathrm{~mm}$ gap, 40 near-axial slices, aligned with the anteriorposterior commissure line). Three hundred image volumes were acquired during the MID task.

Data analysis voxel-based morphometry (VBM). Anatomical data were processed by means of the VBM8 toolbox (http:// dbm.neuro.uni-jena.de/vbm.html) with default parameters by Gaser and the SPM8 software package (http://www.fil.ion.ucl. ac.uk/spm). The VBM8 toolbox involves bias correction, tissue classification and affine registration. The affine registered grey matter (GM) and white matter (WM) segmentations were used to build a customized DARTEL (diffeomorphic anatomical registration through exponentiated lie algebra ${ }^{16}$ ) template. Then warped GM and WM segments were created. Modulation was applied in order to preserve the volume of a particular tissue within a voxel by multiplying voxel values in the segmented images by the Jacobian determinants derived from the spatial normalization step. In effect, the analysis of modulated data tests for regional differences in the absolute amount (volume) of GM. Finally, images were smoothed with a full-width half-maximum kernel of $8 \mathrm{~mm}$. Statistical analysis was carried out by means of whole-brain comparison of GM volume between frequent (more than $9 \mathrm{~h}$ per week) and infrequent video game players (less or equal to $9 \mathrm{~h}$ per week). Sex, scanner and whole brain volume were entered as covariates of no interest. The resulting maps were thresholded with $P<0.001$ and the statistical extent threshold was corrected for multiple comparisons and combined with a nonstationary smoothness correction. ${ }^{17}$

Data analysis fMRI. Preprocessing of the $\mathrm{fMRI}$ data was performed using SPM 8 and comprised slice-timing correction, spatial realignment to the first volume and nonlinear warping to $\mathrm{MNI}$ space. Images were then smoothed with a Gaussian kernel of $5-\mathrm{mm}$ full-width halfmaximum. The model contained the onset of each cue and each feedback presentation, to enable separate analyses of reward anticipation and reward feedback conditions. Each trial was convolved with a hemodynamic response function and movement parameters were included in the design matrix. For the current analyses, we were interested in the contrast comparing feedback of any kind of loss (small or large loss) with feedback of no loss according to the findings of Linnet et al. ${ }^{10}$ We performed a second-level analysis comparing frequent and infrequent video gamers controlling for the nuisance variables sex and scanner. The resulting $t$-maps were initially thresholded with $P<0.001$ and cluster size of 10; small volume correction within the region of structural change in the ventral striatum allowed for familywise error correction with a threshold of $P<0.05$

\section{Results}

Participants played on average $1.5 \mathrm{~h}$ (s.d.=1.8) during regular weekdays and $2.3 \mathrm{~h}(\mathrm{~s} . \mathrm{d} .=2.6$ ) on days during the weekend, in total $12.1 \mathrm{~h}$ per week. When splitting the sample according to weekly hours of video game playing into frequent ( $n=76$ : 24 females, 52 males) and infrequent $(n=78: 58$ females, 20 males) gamers (median $9 \mathrm{~h}$ ) and contrasting GM and WM segmentations between both groups, we found significant higher left ventral striatum GM for frequent vs infrequent video gamers $(P<0.001$, corrected for multiple comparisons; MNI coordinate: $-9,12,-5$; Figure 1a). In order to ensure that the observed effect in ventral striatum was not driven by the different scanners, we repeated the analysis for the two scanners separately. In line with the reported results, we found increases in left ventral striatum (and no additional regions) in frequent compared with infrequent gamers (results in Supplementary Material). No region showed higher GM volume in infrequent compared with frequent video gamers and no significant differences were found in WM segmentations. In order to characterize further functional involvement of the region of higher ventral striatal GM volume, we correlated it with behavioural measures of the CGT. A significant negative correlation between deliberation time and left striatal GM volume $(r(153)=-0.22, P<0.01$, Bonferroni corrected at $P<0.05$, Figure 2) was observed indicating that participants with higher GM volume in ventral striatum were faster in decision making. We analysed brain activity acquired in context of a reward task (MID) and found higher activity in

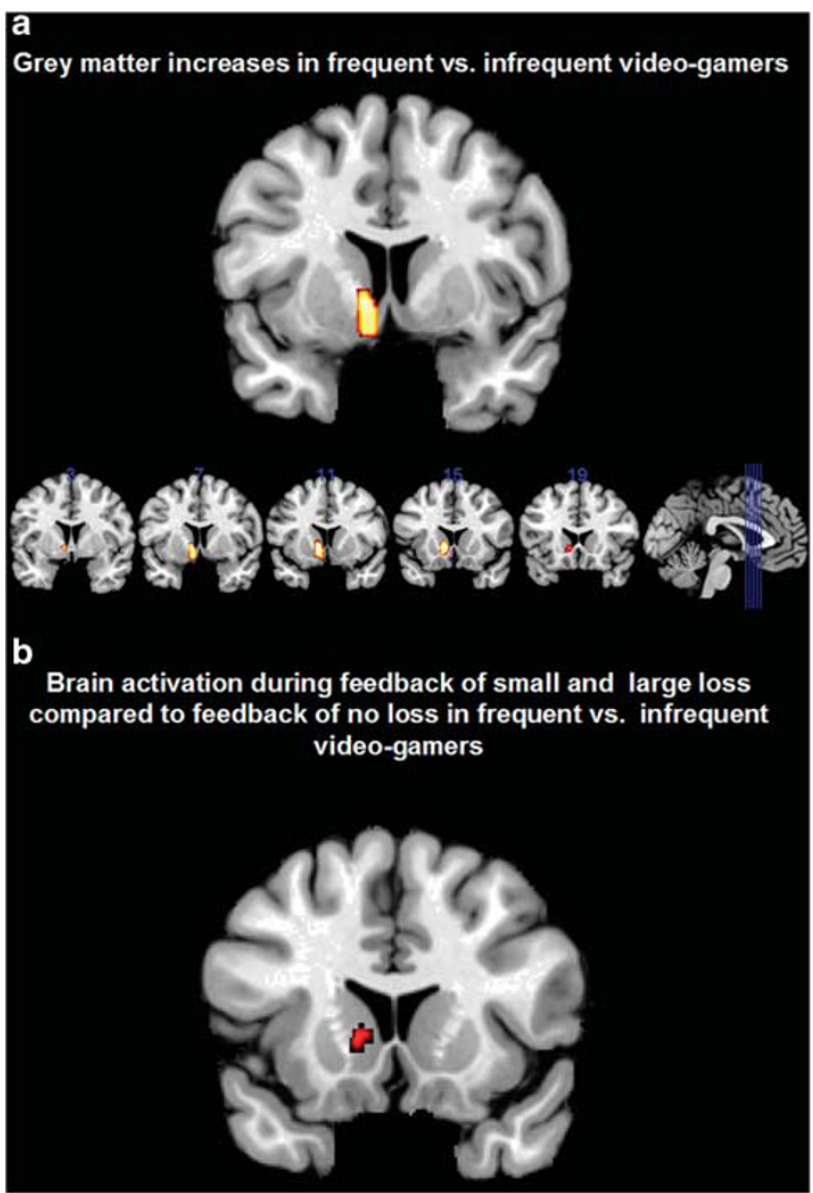

Figure 1 (a) Higher grey matter volume in frequent vs infrequent video game players in left ventral striatum, (b) higher blood oxygen-level-dependent activity in frequent vs infrequent video game players during feedback of small or large loss compared with feedback of no loss. 

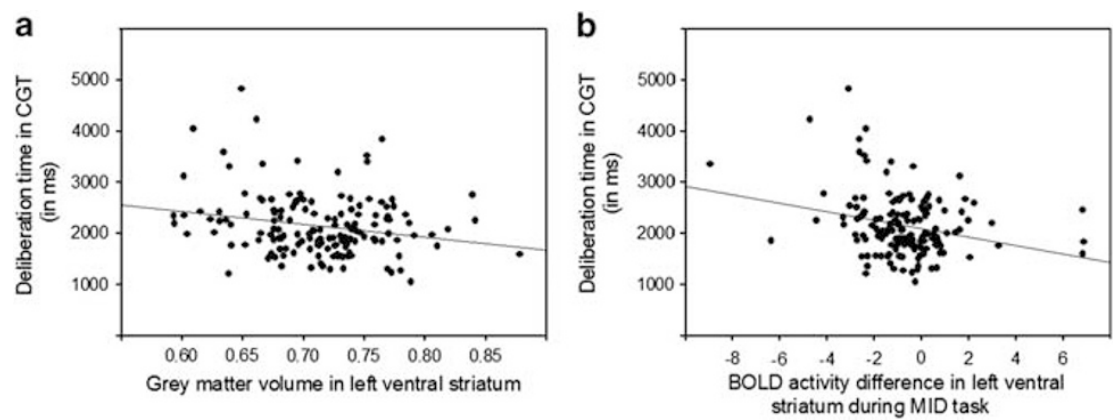

Figure 2 Scatter plot displaying the negative correlation between deliberation time in the Cambridge Gambling Task (CGT) and (a) grey matter volume in left ventral striatum and (b) blood oxygen-level-dependent (BOLD) signal difference between feedback of loss vs feedback of no loss in the Monetary Incentive Delay (MID) task.

frequent compared with infrequent video game players during feedback of loss (small and large) vs feedback of no loss in the MID task overlapping with the region in which we observed higher striatal GM volume $(P<0.001$, uncorrected; for small volume correction in structural cluster of ventral striatum familywise error $P<0.05$; MNI coordinate: -9 , 8, 4; Figure 1b). In analogy to the negative association between deliberation time in CGT and left ventral striatum volume, we found a negative correlation between deliberation time and feedback of loss- vs no-loss-related activation in the MID task $(r(153)=-0.25$, $P<0.01$, Bonferroni corrected at $P<0.05$ ).

\section{Discussion}

The key finding of higher volume in left ventral striatum associated with frequent video game playing is in conceptual accordance with findings of enhanced dopamine release during video game playing ${ }^{5}$ and excessive gambling in Parkinson's patients due to dopaminergic medication. ${ }^{8}$ Striatal release of dopamine as measured in positron emission tomography has been shown to correlate with BOLD response in the striatum, ${ }^{18}$ and therefore suggests a neurochemical link to $\mathrm{fMRI}$ findings that report an association between gambling tasks and BOLD activity in the striatum. ${ }^{6}$ In addition, striatal BOLD activity is predicted by genetic variants of the dopamine system. ${ }^{19,20}$ Whether the volumetric differences in ventral striatum between frequent and moderate video game players are preconditions that lead to a vulnerability for preoccupation with gaming or whether they are a consequence of long-lasting activation during gaming can not be determined with a cross-sectional study. Two previous studies on skill acquisition in video games rather suggest an important role of the striatum in preconditions of frequent video gaming. Erickson et al. $^{21}$ have found a correlation between the volume of dorsal striatum and later training success in a video game. In line with this, Vo et al. ${ }^{7}$ have described an association between pre-training $\mathrm{fMRI}$ activation in the striatum and later skill acquisition during video gaming. These findings suggest the importance of striatal volume and activity in shaping preferences of skills for video gaming rather than striatal changes being the consequence of excessive gaming. Individuals with higher ventral striatum volume might experience video gaming as more rewarding in the first place. This in turn could facilitate skill acquisition and lead to further reward resulting from playing.
Although we did not explicitly explore differences between pathological and non-pathological gaming, volumetric differences in the striatum have previously been associated with addiction to drugs, such as cocaine, ${ }^{22}$ metamphetamine ${ }^{23}$ and alcohol. ${ }^{24}$ However, the direction of the reported differences is not unequivocal; some studies report addiction associated increases others report reductions of the striatal volume most likely due to neurotoxic effects of some drugs of abuse. $^{24}$ If the striatal differences observed in the current study are indeed an effect of gaming, video gaming might pose an interesting option to explore structural changes in addiction in future studies in the absence of any neurotoxic substances.

In order to functionally characterize the volumetric difference observed, we compared BOLD activity between frequent and infrequent video gamers during the feedback of loss compared with the feedback of no loss in the MID task. We found higher activity in frequent compared with infrequent players. Activation in the ventral striatum has been associated with the anticipation and feedback of reward. ${ }^{25}$ In pathological gamblers, an increase in dopamine release in the ventral striatum was found when losing money. ${ }^{10}$ Such a dopaminergic response may attribute incentive salience to gamblingassociated cues ${ }^{26}$ and may explain the so-called 'loss chasing' behaviour during which pathological gamblers continue to gamble despite losing.

The structural and functional results were referenced to performance measures of a behavioural gambling task, which was administered outside the scanner. A significant negative association between deliberation time in placing bets and volume of the ventral striatum as well as functional activity during feedback of loss vs feedback of no loss in ventral striatum was found. This suggests that striatal volume as well as striatal function mediates behavioural measures in gambling. Moreover, a recent study has associated fMRI activity of the striatum (in particular caudate nucleus) with quick generation of the next best move in professional players of a Japanese board game. ${ }^{27}$ Furthermore, short decision times in a gambling task reduce the delay until feedback and expected reward is received and might therefore be facilitated and contribute to an overactive reward network. In neuroimaging studies, exploring the speed-accuracy trade-off striatal activity has been related to criterion setting. ${ }^{28,29}$ In particular, anatomically stronger cortico-striatal connections seem to be associated with the ability to flexibly change response 
thresholds, which might either lead to cautious or more risky behaviour. ${ }^{30}$ Therefore, changes in striatal volume might interact with criterion setting in decision making

Our results have implications for the understanding of the structural and functional basis of excessive but non-pathological video game playing and the role of the ventral striatum in 'behavioral' addiction. They suggest that frequent video game playing is associated with higher volume in left ventral striatum, which in turn shows higher activity during feedback of loss compared with feedback of the absence of loss in frequent players. A negative correlation between deliberation time in betting and GM volume as well as functional activation during feedback of loss in left ventral striatum underlines its functional involvement in gambling-related decision making.

\section{Conflict of interest}

The authors declare no conflict of interest.

Acknowledgements. The IMAGEN study receives research funding from the European Community's Sixth Framework Program (LSHM-CT-2007-037286) and is supported by the UK Department of Health NIHR-Biomedical Research Centre 'Mental Health' and the MRC program grant 'Developmental pathways into adolescents' substance abuse'. Additional funding was provided by the Berliner Senatsverwaltung 'Implikationen biopsychosozialer Grundlagen der Spielsucht für Prävention und Therapie' Vergabe-Nr. 002-2008/ I B 35.

1. Green CS, Bavelier D. Video-game modifies visual selective attention. Nature 2003; 423 534-537

2. Li R, Polat U, Makous W, Bavelier D. Enhancing the contrast sensitivity function through action video-game training. Nat Neurosci 2009; 12: 549-551.

3. Green CS, Pouget A, Bavelier D. Improved probabilistic inferences as a general learning mechanism with action video-games. Curr Biol 2010; 20: 1573-1579.

4. Basak C, Boot WR, Voss MW, Kramer AF. Can training in a real-time strategy video game attenuate cognitive decline in older adults? Psychol Aging 2008; 23: 765-777.

5. Koepp MJ, Gunn RN, Lawrence AD, Cunningham VJ, Dagher A, Jones T et al. Evidence for striatal dopamine release during a video-game. Nature 1998; 393: 266-268.

6. Li X, Lu ZL, D'Argembeau A, Ng M, Bechara A. The lowa gambling task in fMRI images. Hum Brain Mapp 2010; 31: 410-423.

7. Vo LTK, Walther DB, Kramer AF, Erickson KI, Boot WR, Voss MW et al. Predicting individuals' learning success from patterns of pre-learning MRI activity. PLOS ONE 2011; 6: e16093.

8. Dagher A, Robbins TW. Personality, addiction, dopamine: insights from Parkinson's Disease. Neuron 2009; 61: 502-510.

9. Steeves TDL, Miyasaki J, Zurowski M, Lang AE, Pellecchia G, Van Eimeren T et al. Increased Striatal dopamine release in Parkinsonian patients with pathological gambling: a $\left[{ }^{11} \mathrm{C}\right]$ raclopride PET study. Brain 2009; 132: 1376-1385.

10. Linnet J, Peterson E, Doudet DJ, Gjedde A, Moller A. Dopamine release in ventral striatum of pathological gamblers losing money. Acta Psychiatr Scand 2010; 112: 326-333.

11. Schumann $G$, Loth $E$, Banaschewski T, Barbot A, Barker G, Büchel $C$ et al. The IMAGEN study: reinforcement-related behaviour in normal brain function and psychopathology. Mol Psychiatry 2010; 15: 1128-1239.
12. Knutson B, Fong GW, Adams CM, Varner JL, Hommer D. Dissociation of reward anticipation and outcome with event-related fMRI. Neuroreport 2001; 12: 3683-3687.

13. Rogers RD, Everitt BJ, Baldacchino A, Blackshaw AJ, Swainson R, Wynne K. Dissociable deficits in the decision-making cognition of chronic amphetamine abusers, opiate abusers, patients with focal damage to prefrontal cortex, and tryptophan-depleted normal volunteers: evidence for monoaminergic mechanisms. Neuropsychopharmacology 1999; 20: $322-339$.

14. Goodman R, Ford T, Richards H, Gatward R, Meltzer H. The Development and Well-Being Assessment: description and initial validation of an integrated assessment of child and adolescent psychopathology. J Child Psychol Psychiatry 2000; 41: 645-655.

15. Wölfling K, Müller KW, Beutel M. Reliability and validity of the scale for the assessment of pathological computer-gaming (CSV-S). Psychother Psychosom Med Psychol 2011; 61: 216-224.

16. Ashburner J. A fast diffeomorphic image registration algorithm. Neurolmage 2007; 38 : 95-113.

17. Hayasaka S, Nichols TE. Combining voxel intensity and cluster extent with permutation test framework. Neurolmage 2004; 23: 54-63.

18. Schott BH, Minuzzi L, Krebs RM, Elmenhorst D, Lang M, Winz $\mathrm{OH}$ et al. Mesolimbic functional magnetic resonance imaging activations during reward anticipation correlate with reward-related ventral Striatal dopamine release. J Neurosci 2008; 28: 14311-14319.

19. Schmack K, Schlagenhauf F, Sterzer P, Wrase J, Beck A, Dembler T et al. Catechol-Omethyltransferase val158met genotype influences neural processing of reward anticipation. Neuroimage 2008; 42: 1631-1638.

20. Yacubian J, Sommer T, Schroeder K, Gläscher J, Kalisch R, Leuenberger B et al. Gene-gene interaction associated with neural reward sensitivity. Proc Natl Acad Sci USA 2007; 104: 8125-8130.

21. Erickson KI, Boot WR, Basak C, Neider MB, Prakash RS, Voss MW et al. Striatal volume predicts level of video-game skill acquisition. Cerebral Cortex 2010; 20: 2522-2530.

22. Jacobsen LK, Giedd JN, Gottschalk C, Kosten TR, Krystal JH. Quantitative morphology of the caudate and putamen in patients with cocaine dependence. Am J Psychiatry 2001; 158: $486-489$.

23. Chang L, Alicata D, Ernst T, Volkow N. Structural and metabolic brain changes in the striatum associated with metamphetamine abuse. Addiction 2007; 102(Suppl 1): 16-32.

24. Wrase J, Makris N, Braus DF, Mann K, Smolka MN, Kennedy DN et al. Amygdala volume associated with alcohol abuse relapse and craving. Am J Psychiatry 2008; 165: 1179-1184.

25. Schlagenhauf F, Sterzer P, Schmack K, Ballmaier M, Rapp M, Wrase J et al. Reward feedback alterations in unmedicated schizophrenia patients: relevance for delusions. Biol Psychiatry 2009; 65: 1032-1039.

26. Wrase J, Grüsser SM, Klein S, Diener C, Hermann D, Flor H et al. Development of alcoholassociated cues and cue-induced brain activation in alcoholics. Eur Psychiatry 2002; 17: 287-291

27. Wan X, Nakatani H, Ueno K, Asamizuya T, Cheng K, Tanaka K. The neural basis of intuitive best next-move generation in board game experts. Science 2011; 21: 341-346.

28. Bogacz R, Wagenmakers E-J, Forstmann BU, Nieuwenhuis S. The neural basis of the speed-accuracy trade-off. Trends Neurosci 2010; 33: 10-16.

29. Kühn S, Schmiedek F, Schott B, Ratcliff R, Heinze HJ, Düzel E et al. Brain areas consistently linked to individual differences in perceptual decision-making in younger as well as older adults before and after training. J Cogn Neurosci 2011; 23: 2147-2158.

30. Forstmann BU, Anwander A, Schäfer A, Neumann J, Brown S, Wagenmakers EJ et al. Cortico-striatal connections predict control over speed and accuracy in perceptual decision making. Proc Natl Acad Sci USA 2010; 107: 15916-15920.

Translational Psychiatry is an open-access journal published by Nature Publishing Group. This work is licensed under the Creative Commons Attribution-Noncommercial-No Derivative Works 3.0 Unported License. To view a copy of this license, visit http://creativecommons.org/licenses/by-nc-nd/3.0/

Supplementary Information accompanies the paper on the Translational Psychiatry website (http://www.nature.com/tp) 